\title{
Electronic Configuration of Yb Compounds
}

\author{
W. M. Temmerman, ${ }^{1}$ Z. Szotek, ${ }^{1}$ A. Svane, ${ }^{2}$ P. Strange, ${ }^{3}$ H. Winter, ${ }^{4}$ A. Delin, ${ }^{5}$ B. Johansson, ${ }^{5}$ O. Eriksson, ${ }^{5}$ \\ L. Fast, ${ }^{5}$ and J.M. Wills ${ }^{6}$ \\ ${ }^{1}$ Daresbury Laboratory, Daresbury, Warrington, WA4 4AD, Cheshire, United Kingdom \\ ${ }^{2}$ Institute of Physics and Astronomy, University of Aarhus, DK-8000 Aarhus C, Denmark \\ ${ }^{3}$ Physics Department, Keele University, Keele, Staffordshire, ST5 5BG, United Kingdom \\ ${ }^{4}$ INFP, Forschungszentrum Karlsruhe GmbH, Postfach 3640, D-76021 Karlsruhe, Germany \\ ${ }^{5}$ Condensed Matter Theory Group, Department of Physics, University of Uppsala, Box 530, 75121 Uppsala, Sweden \\ ${ }^{6}$ Center of Materials Science and Theoretical Division, Los Alamos National Laboratory, Los Alamos, New Mexico 87544
}

(Received 25 June 1999)

The total energy differences between divalent and trivalent configurations of $\mathrm{Yb}$ ions in a number of $\mathrm{Yb}$ compounds are studied. Two different band theoretical methods, which differ in the treatment of the localized $f$ electrons, are used. The results show that in all $\mathrm{Yb}$ compounds the valence energy differences are equal to the energy needed to localize an $f$ electron. These valence energy differences correlate with the number of $f$ electrons hybridizing with the conduction bands in the trivalent configuration. For divalent $\mathrm{YbS}$, the pressure induced $f$-electron delocalization implies an intermediate valency, as also indicated by experiment.

PACS numbers: 71.15.Nc, 71.20.Eh

The most intriguing aspect of the physics of the lanthanides is possibly connected to the stability of different electronic configurations. Most of the lanthanide elements have three chemically bonding electrons which form a rather wide spd band, but for $\mathrm{Eu}$ and $\mathrm{Yb}$ it is favorable to remove one of these electrons and add it to the $f$ shell. Experimentally there are hence two pertinent electronic configurations (valence stabilities): $4 f^{n}[s p d]^{3}$ and $4 f^{n+1}[s p d]^{2}$. Most of the observed physical and chemical properties of the lanthanides are then characteristic for, respectively, trivalent and divalent electron systems. The situation becomes more intricate when one considers surfaces or compounds which contain lanthanides. Often one can, by forming a compound, change the valence stability, and a lanthanide which is divalent as an element may be in a trivalent configuration in a compound. An interesting class of materials displaying this behavior is the $\mathrm{Yb}$ monopnictides [1], and here we have chosen to study these and other $\mathrm{Yb}$ compounds.

Because of the interesting properties which are associated with the question of valency of the lanthanides (for instance, mixed and intermediate valency and heavy fermion behavior), it is important to have a good theoretical understanding of what stabilizes a given electronic configuration. In particular, it is of interest to have a theoretical tool which reliably determines the valence stability of any lanthanide system, since it may not always be possible to infer this reliably from experiment. An example is the pressure-volume measurements for $\mathrm{YbS}$ which indicate anomalous behavior above $100 \mathrm{kbar}$ which could be associated with intermediate valency [2]. A trivalent state cannot seemingly be realized for this system. In this case, our study will shed light on properties of this intermediate valence state.
To obtain insight into the mechanisms that determine the valence stability, in this Letter we apply two different approaches to study the energy differences between various valence configurations in $\mathrm{Yb}$ compounds. Both approaches are based on $a b$ initio band structure methods, in which the $\mathrm{Yb}$ ions are constrained to be in either $f^{14}$ (divalent) or $f^{13}$ (trivalent) configuration. In the first approach [3], which combines a density functional, solid state, method with input from experimental atomic data, a particular configuration is accomplished by, respectively, placing either 14 or $13 f$ electrons in the core. This approach recognizes the fact that density functional theory calculations, which rely on the local density approximation or gradient expansions, cannot reproduce all coupling energies of atomiclike open electron shells. However, these energies may be extracted from experiments and one can calculate the total energy of such a system by first calculating the energy of a constrained density functional, where the $f$ occupation is fixed and the hybridization between the $f$ states and all other states is forced to be zero. To this constrained energy expression one then adds the experimental atomic multiplet energy. This method, to which we shall refer to as the "combined approach," was successfully applied to the elemental rare earth metals, demonstrating that the energetics of the $f^{n}$ shell in the solid are well accounted for by the corresponding free atomic numbers. As a particularly sensitive test the intricate valence stability of Sm and Tm chalcogenides was reproduced [3]. The second approach [4] is ab initio and solely solid state based. It has the advantage of being able to describe the localization of an electron through the consideration of the self-interaction correction (SIC) to the local-spindensity (LSD) approximation, for either 14 or $13 f$ electrons, respectively, in divalent $\left(\mathrm{Yb}^{2+}\right)$ and trivalent $\left(\mathrm{Yb}^{3+}\right)$ 
ionic configurations. In this SIC-LSD approach all electrons are treated as bandlike, but when an $f$ electron is localized it experiences a potential which is corrected for self-interaction, while an itinerant (fast-moving) electron moves in a mean-field potential, as given by the LSD approximation. Our implementation of the SIC-LSD formalism within the linear-muffin-tin-orbital method [5] is discussed in detail in Ref. [4]. The SIC-LSD method has been used successfully for describing the electronic and magnetic properties of rare earths [6].

The combined approach enforces an integer valence change by putting either $n$ or $n+1 f$ electrons into the core, thus strictly imposing the presence of 2 and 3 non- $f$-valence electrons in the divalent and trivalent configurations, respectively. In contrast, in the SIC-LSD representation of the trivalent (divalent) state $n(n+1) f$ electrons will be localized and nonbonding, while the remaining $14-n(13-n) f$ electrons are allowed to hybridize with the valence band. This can therefore increase the $f$-electron occupancy beyond $n$ and $n+1$ and reduce the number of non- $f$-valence electrons in comparison with the combined approach. The energy difference between the $n$ and $(n+1)$ configurations may be termed the (SIC-LSD) $f$-electron localization energy. Another way to express this is that the SIC-LSD can describe a gradual or partial delocalization of the $f$ manifold [7]. Similar ideas have been considered in calculations on $\delta$-Pu [8] and in theories starting from the strongly correlated electron picture [9]. Since it is common to analyze pressure induced anomalies of the lattice constant with the changes in valency, the concurrent application of both approaches will verify in a direct manner whether the valency change and the change in $f$-electron localization are always synonymous. In particular, the question can be posed if change in $f$-electron localization can occur without significant change in valency, which could be realized in intermediate valence systems.

The energy differences between the divalent and trivalent solutions, as obtained within the combined approach, are compared in Fig. 1 with the SIC-LSD energy differences between compounds having 14 and 13 localized $\mathrm{Yb}$ $f$ electrons. A positive energy difference in Fig. 1 indicates that the trivalent configuration is more stable than the divalent configuration. The first thing to note is that both methods correctly predict the valency stability of the compounds studied, i.e., $\mathrm{Yb}$ metal and $\mathrm{Yb}$ chalcogenides are found divalent, while all the $\mathrm{Yb}$ monopnictides (including hypothetical $\mathrm{YbBi}$ ) as well as $\mathrm{YbBiPt}$ are trivalent. In general, the agreement between the SIC approach and the combined approach is good, as the plotted points are situated on both sides of the diagonal, which marks a perfect agreement line. This demonstrates that the $f$-electron localization energy determines the energy difference between the divalent and trivalent states. Moreover, the results clearly show the progression from the most distinctly trivalent compound, $\mathrm{YbN}$, through de-

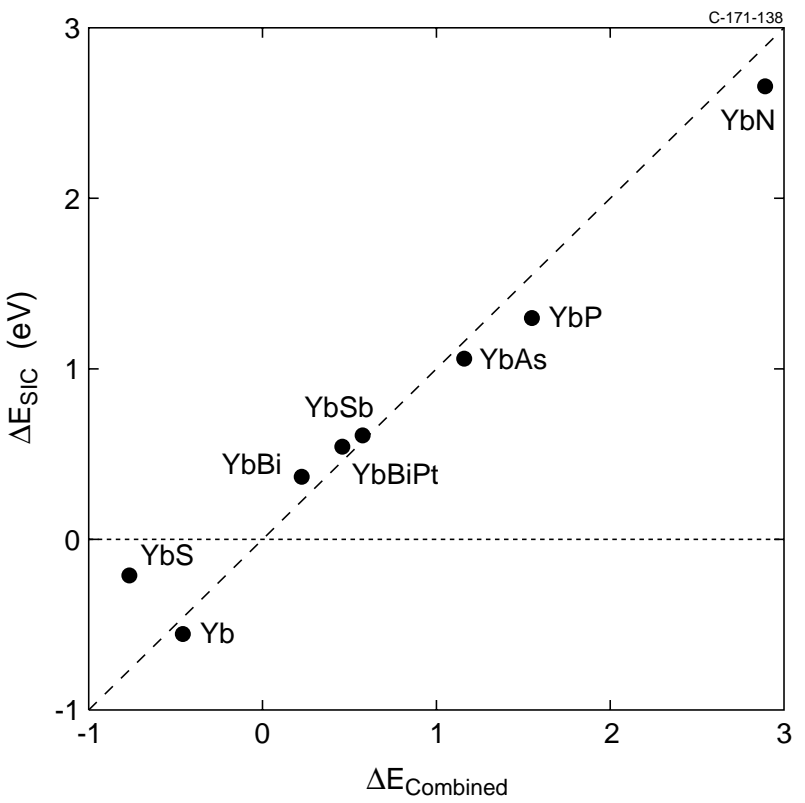

FIG. 1. Comparison of "combined" and SIC-LSD calculations of the energy difference (in $\mathrm{eV}$ ) between $\mathrm{Yb}$ compounds having a divalent and a trivalent $\mathrm{Yb}$ configuration. A positive energy difference means that the trivalent configuration is the ground state. The SIC calculations include a spin-orbit correction of $+0.54 \mathrm{eV}$.

creasing trivalency with an increasing atomic number of the pnictide in $\mathrm{YbP}$ and $\mathrm{YbAs}$, over the weakly trivalent $\mathrm{YbSb}, \mathrm{YbBi}, \mathrm{YbBiPt}$, and $\mathrm{YbPd}$, to the divalent $\mathrm{Yb}, \mathrm{YbS}$, $\mathrm{YbSe}$, and YbTe. The theoretical equilibrium volumes are in all cases found to agree within a few percent with the experimental values [10].

Only for $\mathrm{Yb}$ metal is the energy difference between the trivalent and divalent states known experimentally, $0.50 \mathrm{eV}$ [11], and both approaches are in good agreement with this value. This implies that for elemental $\mathrm{Yb}$, too, valency and localization mean the same thing. Alloying $\mathrm{S}$ into $\mathrm{Yb}$ reduces the stability of the divalent state according to the SIC approach but enhances it in the combined approach. The increase of divalency energy in the combined approach in going from $\mathrm{Yb}$ to $\mathrm{YbS}$ is consistent, in a chemical sense, with what happens in $\mathrm{SmS}$. Elemental $\mathrm{Sm}$ is trivalent, but incorporation of $\mathrm{S}$ in Sm makes this compound divalent.

While both theoretical approaches address the localization of the $f$ states, they differ substantially in the $f$-electron count. As stated above, in the combined approach, the trivalent and divalent calculations have an integer number of $f$ electrons of, respectively, 13 and 14, and they are part of the core. In the SIC approach, on the other hand, the $f$ electrons are not part of the core, and their number is not restricted to integer values. For the systems in the divalent configuration the $f$ occupancy is found close to the value 14, while for the systems in the trivalent configuration the $f$ occupancy varies between 
13 and 14. When trivalency is strongly favored, as in $\mathrm{YbN}$, we find an $f$ occupancy closer to 13 than 14, while the systems where divalency is favored, as YbTe, have in their trivalent configuration an $f$ occupancy closer to 14 than 13. In Fig. 2, we show for $\mathrm{Yb}$ compounds the SIC total $f$-occupancy difference between the divalent and trivalent configurations versus their energy difference. The relation is close to linear. The largest $f$-occupancy difference, 0.8 electrons, occurs in $\mathrm{YbN}$, for which we also obtain the highest energy difference between the two electronic configurations. This electron difference gradually decreases to approximately 0.5 for $\mathrm{YbPd}, \mathrm{YbSb}, \mathrm{YbBi}$, and $\mathrm{YbBiPt}$, and for the four divalent Yb systems we obtain between 0.22 and 0.35 electrons. The substantially reduced difference in $f$-electron count seems to herald the arrival of divalent behavior. The divalent $\mathrm{YbS}, \mathrm{YbSe}$, and $\mathrm{YbTe}$ are characterized by semiconducting behavior as a consequence of a full $f$ shell and a full chalcogenide $p$ band. The gaps calculated at ambient conditions within the SIC approach are 2.2, 1.8 , and $1.5 \mathrm{eV}$, respectively, which compare favorably with the experimental absorption edges of 1.8, 1.5, and $1.1 \mathrm{eV}$ [12]. The $\mathrm{YbS}$ gap in the combined approach is likewise $2.2 \mathrm{eV}$. In trivalent $\mathrm{YbS}$ the unoccupied $f$ peak hybridizes strongly with the $s p d$ electrons, to such an extent that only $0.3 f$ electrons less are occupied than in the divalent case. Actually the hybridization details of this unoccupied $f$ state determine how much deviation from "trivalency" occurs. Ideal trivalency would mean this single $f$ state to be fully unoccupied with no hybridization with the $d$ states. Any deviation from

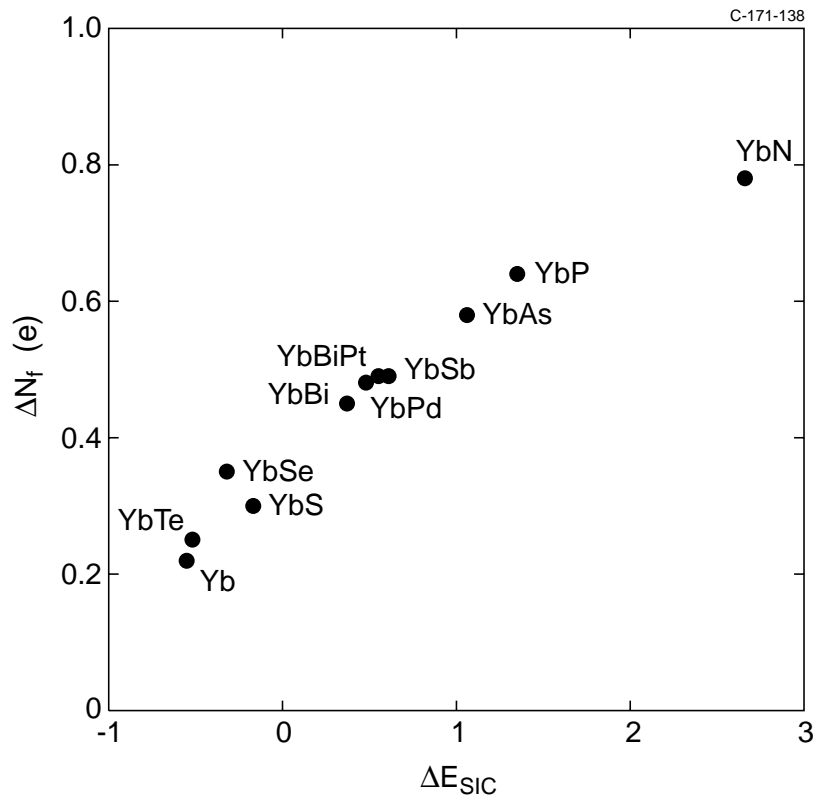

FIG. 2. Divalent-trivalent energy difference (in $\mathrm{eV}$ ) of $\mathrm{Yb}$ compounds versus the total $f$-occupancy difference in the divalent and trivalent configurations, as given by the SIC-LSD approach. this means intermediate valency. In the strongly trivalent $\mathrm{Yb}$ compounds, such as $\mathrm{YbN}$ and $\mathrm{YbP}$, the unoccupied $f$ peak is further removed from the Fermi level, and the small additional $f$ occupancy reflects the hybridization with the $p$ band. In the weakly trivalent and the divalent $\mathrm{Yb}$ compounds this unoccupied $f$ peak is moved closer to the Fermi level. The fact that $\mathrm{YbN}$ deviates a little from linearity in Fig. 2 may be due to a slight overestimation of this hybridization in our calculations.

In Fig. 3 the pressure-volume curve for YbS as calculated in the SIC approach is shown and compared to the experimental data [2]. At pressures below $100 \mathrm{kbar}$ the experimental and theoretical (14 localized $f$ electrons) curves coincide. Above $100 \mathrm{kbar}$ the experimental curve is clearly anomalous indicating valence instability [2]. From the common tangent construction of the total energies as a function of volume for both divalent and trivalent $\mathrm{YbS}$, we obtain with the SIC approach a transition pressure that is $\sim 75 \mathrm{kbar}$ and agrees well with the observed onset of anomalous behavior around $\sim 100 \mathrm{kbar}$. The combined approach overestimates this transition pressure somewhat, providing a value of about $230 \mathrm{kbar}$. This suggests that the experimental anomaly is not really correlated with an integer change in valency but is as a matter of fact due to a partial $f$-electron delocalization. The quantitative theoretical description of this seemingly continuous valence transition calls for a more elaborate theory than presented here. The small change in $f$-electron occupancy of 0.3 electrons found with the SIC approach upon delocalization of an $f$ electron suggests the occurrence of intermediate valency of 2.3. This is consistent with the experimental estimate of an intermediate valency of 2.4 [2].

The heavy fermion system YbBiPt is trivalent in both approaches. Energetically this system is $0.5 \mathrm{eV}$ away from divalency. This rules out mixed valency to be associated with the heavy fermion behavior of this system. However, the SIC study indicates a substantial $f$ - $(s p d)$

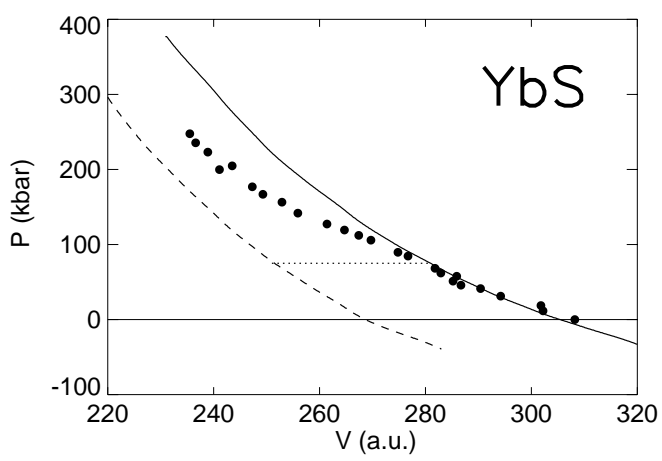

FIG. 3. Equation of state for $\mathrm{YbS}$ as calculated by the SIC-LSD method. The two theoretical curves correspond to 14 (solid line) and 13 (dashed line) localized $f$ electrons, respectively, while the dots are the experimental data of Ref. [2]. The dotted line marks the theoretical transition. 
hybridization leading to an $f$ occupancy nearly halfway between divalent and trivalent states, suggesting intermediate valency. The density of states at the Fermi energy is large, and the value of the spin magnetic moment is $0.5 \mu_{B}$. These findings are consistent with those obtained by the LDA $+U$ method for this compound [13]. YbPd experimentally shows a signature of mixed valency [14], and by the SIC-LSD theory this compound, like YbBiPt, is predicted to be intermediate between divalent and trivalent, albeit weakly trivalent.

In conclusion, we have identifed theoretically divalent, trivalent, and intermediate valency in $\mathrm{Yb}$ compounds. By applying two different methodologies we have been able to shed light on the relation between the valency and localization energy of $f$ electrons. Specifically, from the SIC calculations we have been able to establish that a change in valency is sometimes associated with a partial $f$-electron delocalization, i.e., part of the $f$ shell experiences a stronger tendency to localize, resulting in bands with little dispersion, while other $f$-derived states are more delocalized and have more dispersion. This unusual behavior of the $f$ electrons is well illustrated by the presently studied compounds, where $\mathrm{YbN}$ behaves mostly as one would expect from an ionic picture of a trivalent system, whereas all other systems show varying degrees of intermediate valency behavior. The study of divalent YbS demonstrates that $f$-electron delocalization does not always imply integer valency change and we found $\mathrm{YbS}$ under pressure to be of intermediate valency character.

This work has benefited from collaborations within and has been partially funded by the Training and Mobility Network on "Electronic structure calculations of materials properties and processes for industry and basic sciences" (Contract No. FMRX-CT98-0178).
[1] G.H. Dieke, Spectra and Energy Levels of Rare Earth Ions in Crystals (Wiley, New York, 1968).

[2] K. Syassen, H. Winzen, H. G. Zimmer, H. Tups, and J. M. Leger, Phys. Rev. B 32, 8246 (1985).

[3] A. Delin, L. Fast, B. Johansson, J.M. Wills, and O. Eriksson, Phys. Rev. Lett. 79, 4637 (1997); A. Delin, L. Fast, B. Johansson, O. Eriksson, and J. M. Wills, Phys. Rev. B 58, 4345 (1998).

[4] W. M. Temmerman, A. Svane, Z. Szotek, and H. Winter, in Electronic Density Functional Theory: Recent Progress and New Directions, edited by J. F. Dobson, G. Vignale, and M.P. Das (Plenum, New York, 1998).

[5] O. K. Andersen, Phys. Rev. B 12, 3060 (1975).

[6] I. V. Solov'ev, A.I. Liechtenstein, and V.A. Gubanov, Sov. Phys. Solid State 33, 572 (1991); Z. Szotek, W. M. Temmerman, and H. Winter, Phys. Rev. Lett. 72, 1244 (1994); A. Svane, Phys. Rev. Lett. 72, 1248 (1994); R. Schumann, M. Richter, L. Steinbeck, and H. Eschrig, Phys. Rev. B 52, 8801 (1995); A. Svane, Z. Szotek, W. M. Temmerman, J. Lægsgaard, and H. Winter, J. Phys. Condens. Matter 10, 5309 (1998); P. Strange, A. Svane, W.M. Temmerman, Z. Szotek, and H. Winter, Nature (London) 399, 756 (1999).

[7] W. M. Temmerman, Z. Szotek, and H. Winter, Phys. Rev. B 47, 1184 (1993).

[8] O. Eriksson, J.D. Becker, A. V. Balatsky, and J.M. Wills, J. Alloys Compd. 287, 1 (1999).

[9] U. Lundin, I. Sandalov, O. Eriksson, and B. Johansson, Physica (Amsterdam) 230B-232B, 445 (1997).

[10] A. Svane et al. (unpublished).

[11] B. Johansson, Phys. Rev. B 20, 1315 (1979).

[12] V. Narayanamurti, A. Jayaraman, and E. Bucher, Phys. Rev. B 9, 2521 (1974).

[13] P. Oppeneer, V.N. Antonov, A.N. Yaresko, A. Ya. Perlov, and H. Eschrig, Phys. Rev. Lett. 78, 4079 (1997).

[14] P. Bonville, J. Hammann, J. A. Hodges, P. Imbert, and G. J. Jéhanno, Phys. Rev. Lett. 57, 2733 (1986). 